\title{
A Note on the Cosmological Constant in $f(R)$ Gravity
}

\author{
Peter K. F. Kuhfittig \\ Department of Mathematics, Milwaukee School of Engineering, Milwaukee, Wisconsin, USA \\ Email:kuhfitti@msoe.edu
}

How to cite this paper: Kuhfittig, P.K.F. (2017) A Note on the Cosmological Constant in $f(R)$ Gravity. Journal of Applied Mathematics and Physics, 5, 933-938. https://doi.org/10.4236/jamp.2017.54082

Received: March 14, 2017

Accepted: April 27, 2017

Published: April 30, 2017

Copyright (c) 2017 by author and Scientific Research Publishing Inc. This work is licensed under the Creative Commons Attribution International License (CC BY 4.0).

http://creativecommons.org/licenses/by/4.0/

\begin{abstract}
The starting point in this note is $f(R)$ modified gravity in a cosmological setting. We assume a spatially flat universe to describe late-time cosmology and the perfect-fluid equation of state $p=\omega \rho$ to model the hypothesized dark energy. Given that on a cosmological scale, $f(R)$ modified gravity must remain close to Einstein gravity to be consistent with observation, it was concluded that either (1) Einstein's cosmological constant was the only acceptable model for the accelerated expansion, or that (2) the equation of state for dark energy was far more complicated than the perfect-fluid model and might even exclude a constant $\omega$.
\end{abstract}

\section{Keywords}

Cosmological Constant, $f(R)$ Gravity

\section{Introduction}

The discovery that our universe is undergoing an accelerated expansion [1] [2] has led to a renewed interest in modified theories of gravity. One of the most important of these, $f(R)$ modified gravity, replaces the Ricci scalar $R$ in the Einstein-Hilbert action

$$
S_{\mathrm{EH}}=\int \sqrt{-g} R \mathrm{~d}^{4} x
$$

by a nonlinear function $f(R)$ :

$$
S_{f(R)}=\int \sqrt{-g} f(R) \mathrm{d}^{4} x .
$$

(For a review, see Refs. [3] [4] [5].)

An alternative to the modified gravity model is the hypothesis that the acceleration is due to a negative pressure dark energy, implying that $\ddot{a}>0$ in the 
Friedmann equation

$$
\frac{\ddot{a}(t)}{a(t)}=-\frac{4 \pi}{3}(\rho+3 p) .
$$

(We are using units in which $c=G=1$.) In the equation of state $p=\omega \rho$, $\ddot{a}>0$ corresponds to the range of values $\omega<-1 / 3$, referred to as quintessence dark energy. The case $\omega=-1$ is equivalent to assuming Einstein's cosmological constant. It has been forcefully argued by Bousso [6] that the cosmological constant is the best model for dark energy. In this note, we go a step further and propose that $f(R)$ modified gravity implies that $\omega=-1$ is the only allowed value in the equation of state $p=\omega \rho$.

\section{The Solution}

For convenience of notation, we start with the spherically symmetric line element

$$
\mathrm{d} s^{2}=-\mathrm{e}^{2 \Phi(r)} \mathrm{d} t^{2}+\frac{\mathrm{d} r^{2}}{1-b(r) / r}+r^{2}\left(\mathrm{~d} \theta^{2}+\sin ^{2} \theta \mathrm{d} \phi^{2}\right) .
$$

It was shown by Lobo [7] that under the assumption that $\Phi^{\prime}(r) \equiv 0$, the Einstein field equations are

$$
\begin{gathered}
\rho(r)=F(r) \frac{b^{\prime}(r)}{r^{2}}, \\
p_{r}(r)=-F(r) \frac{b(r)}{r^{3}}+F^{\prime}(r) \frac{r b^{\prime}(r)-b(r)}{2 r^{2}}-F^{\prime \prime}(r)\left(1-\frac{b(r)}{r}\right),
\end{gathered}
$$

and

$$
p_{t}(r)=-\frac{F^{\prime}(r)}{r}\left(1-\frac{b(r)}{r}\right)+\frac{F(r)}{2 r^{3}}\left[b(r)-r b^{\prime}(r)\right],
$$

where $F=\frac{\mathrm{d} f}{\mathrm{~d} R}$. The curvature scalar $R$ is given by

$$
R(r)=\frac{2 b^{\prime}(r)}{r^{2}}
$$

For our purposes, a more convenient form of the line element is

$$
\mathrm{d} s^{2}=-\mathrm{e}^{v(r)} \mathrm{d} t^{2}+\mathrm{e}^{\lambda(r)} \mathrm{d} r^{2}+r^{2}\left(\mathrm{~d} \theta^{2}+\sin ^{2} \theta \mathrm{d} \phi^{2}\right) .
$$

Here the Einstein field equations can be written [8]

$$
\begin{aligned}
& 8 \pi \rho=\mathrm{e}^{-\lambda}\left(\frac{\lambda^{\prime}}{r}-\frac{1}{r^{2}}\right)+\frac{1}{r^{2}}, \\
& 8 \pi p_{r}=\mathrm{e}^{-\lambda}\left(\frac{1}{r^{2}}+\frac{v^{\prime}}{r}\right)-\frac{1}{r^{2}}
\end{aligned}
$$

and

$$
8 \pi p_{t}=\frac{1}{2} \mathrm{e}^{-\lambda}\left[\frac{1}{2}\left(v^{\prime}\right)^{2}+v^{\prime \prime}-\frac{1}{2} \lambda^{\prime} v^{\prime}+\frac{1}{r}\left(v^{\prime}-\lambda^{\prime}\right)\right] .
$$


Then if $v^{\prime} \equiv 0$, Lobo's equations become

$$
\begin{gathered}
8 \pi \rho(r)=F(r)\left[\mathrm{e}^{-\lambda}\left(\frac{\lambda^{\prime}}{r}-\frac{1}{r^{2}}\right)+\frac{1}{r^{2}}\right], \\
8 \pi p_{r}(r)=F(r)\left[\mathrm{e}^{-\lambda} \frac{1}{r^{2}}-\frac{1}{r^{2}}\right]+\frac{F^{\prime}(r)}{2} \lambda^{\prime} \mathrm{e}^{-\lambda}-F^{\prime \prime}(r) \mathrm{e}^{-\lambda}
\end{gathered}
$$

and

$$
8 \pi p_{t}(r)=-\frac{F^{\prime}(r)}{r} \mathrm{e}^{-\lambda}-\frac{F(r)}{2 r} \lambda^{\prime} \mathrm{e}^{-\lambda}
$$

Now substituting into the equation of state $p=\omega \rho$, we obtain

$$
\begin{aligned}
& \omega F(r)\left[\mathrm{e}^{-\lambda}\left(\frac{\lambda^{\prime}}{r}-\frac{1}{r^{2}}\right)+\frac{1}{r^{2}}\right] \\
& =F(r)\left[\frac{\mathrm{e}^{-\lambda}}{r^{2}}-\frac{1}{r^{2}}\right]+\frac{F^{\prime}(r)}{2} \lambda^{\prime} \mathrm{e}^{-\lambda}-F^{\prime \prime}(r) \mathrm{e}^{-\lambda} .
\end{aligned}
$$

This equation can be rewritten as follows:

$$
F^{\prime \prime}(r)-\frac{1}{2} F^{\prime}(r) \lambda^{\prime}+F(r)\left[\omega \frac{\lambda^{\prime}}{r}+(\omega+1) \frac{1}{r^{2}}\left(\mathrm{e}^{\lambda}-1\right)\right]=0 .
$$

Since we are dealing with a cosmological setting, we may assume the FLRW model, so that $v \equiv 0$ :

$$
\mathrm{d} s^{2}=-\mathrm{d} t^{2}+a^{2}(t)\left[\frac{\mathrm{d} r^{2}}{1-k r^{2}}+r^{2}\left(\mathrm{~d} \theta^{2}+\sin ^{2} \theta \mathrm{d} \phi^{2}\right)\right] .
$$

Observe that we now have

$$
\mathrm{e}^{\lambda}=a^{2}(t) \frac{1}{1-k r^{2}}
$$

and

$$
\lambda=\ln a^{2}(t)+\ln \left(1-k r^{2}\right)^{-1},
$$

so that

$$
\lambda^{\prime}=\frac{2 k r}{1-k r^{2}}
$$

which is independent of time. The significance of the special value $\omega=-1$ in Equation (14) now becomes apparent: the entire equation has become time independent, i.e.,

$$
F^{\prime \prime}(r)-\frac{k r}{1-k r^{2}} F^{\prime}-\frac{2 k}{1-k r^{2}} F=0 .
$$

(For later reference, observe that if $k=0$, then $F(r)=c_{1}+c_{2} r$.) The solution of Equation (19) is

$$
\begin{aligned}
F(r)= & c_{1} \cos \left[\sqrt{2} \ln \left(|k| r+\sqrt{k^{2} r^{2}-1}\right)\right] \\
& +c_{2} \sin \left[\sqrt{2} \ln \left(|k| r+\sqrt{k^{2} r^{2}-1}\right)\right], \quad k \neq 0 .
\end{aligned}
$$

This solution can also be written 


$$
F(r)=c \sin \left[\sqrt{2} \ln \left(|k| r+\sqrt{k^{2} r^{2}-1}+\phi\right]\right.
$$

where $c=\sqrt{c_{1}^{2}+c_{2}^{2}}$ and $\phi=\tan ^{-1}\left(c_{1} / c_{2}\right)$.

\section{Staying Close to Einstein Gravity}

In a cosmological setting, $f(R)$ modified gravity must remain close to Einstein gravity to be consistent with observation. In this section, we wish to show that it is possible, at least in principle, to choose the arbitrary constants in Equation (21) so that this goal is achieved.

The sinusoidal solution (21) has a large period and a small slope, especially for large $r$. To confirm this statement, observe that the function

$$
g(r)=\sin \left[\sqrt{2} \ln \left(|k| r+\sqrt{k^{2} r^{2}-1}\right)\right] \sim \sin (\ln r)
$$

for large $r$. So both $g^{\prime}(r)$ and $g^{\prime \prime}(r)$ approach zero as $r \rightarrow \infty$. As a result, $\sin (\ln r)$ has the approximate form $a r+b$ on any interval that is not excessively large, and since the slope $a$ is small in absolute value, we have

$$
a r+b \approx b, \quad-1 \leq b \leq 1 \text {. }
$$

We can now show that it is possible in principle to choose the arbitrary constants $c$ and $\phi$ in such a way that $F(r)$ remains close to unity and both $F^{\prime}$ and $F^{\prime \prime}$ close to zero on one complete period.

Let $\phi=0$, so that

$$
F(r)=c \sin \left[\sqrt{2} \ln \left(|k| r+\sqrt{k^{2} r^{2}-1}\right)\right] .
$$

First observe that $F(r)=0$ whenever

$$
\sqrt{2} \ln \left(|k| r+\sqrt{k^{2} r^{2}-1}\right)=n \pi
$$

for all integers $n$. Solving for $r$, we get

$$
r=\frac{1}{|k|} \cosh \frac{n \pi}{2} \text {. }
$$

Now choose a particular $n$ for which $F(r) \geq 0$ on the interval $\left[r_{1}, r_{2}\right]$, where

$$
r_{1}=\frac{1}{|k|} \cosh \frac{n \pi}{2} \quad \text { and } \quad r_{2}=\frac{1}{|k|} \cosh \frac{(n+1) \pi}{2}
$$

Next, subdivide the interval $\left[r_{1}, r_{2}\right]$ into $i$ subintervals $I_{i}$ each of which is small enough so that $b$ remains in a narrow range. Then on each separate subinterval, construct a tangent line $a_{i} r+b_{i} \approx b_{i}$ near the midpoint, thereby ensuring that $b_{i} \neq 0$. (See Figure 1 ) So we may now choose $c_{i}=1 / b_{i}$ for the arbitrary constant $c$. We then repeat the procedure on the interval $\left[r_{2}, r_{2}+\pi\right]$, so that $F(r) \approx 1$ on the entire period $\left[r_{1}, r_{2}+\pi\right]$. Since both $F^{\prime}$ and $F^{\prime \prime}$ are close to zero [from Equation (22)], the periodicity of $F(r)$ guarantees that our $f(R)$ modified gravity is close to Einstein gravity for all $r$. 


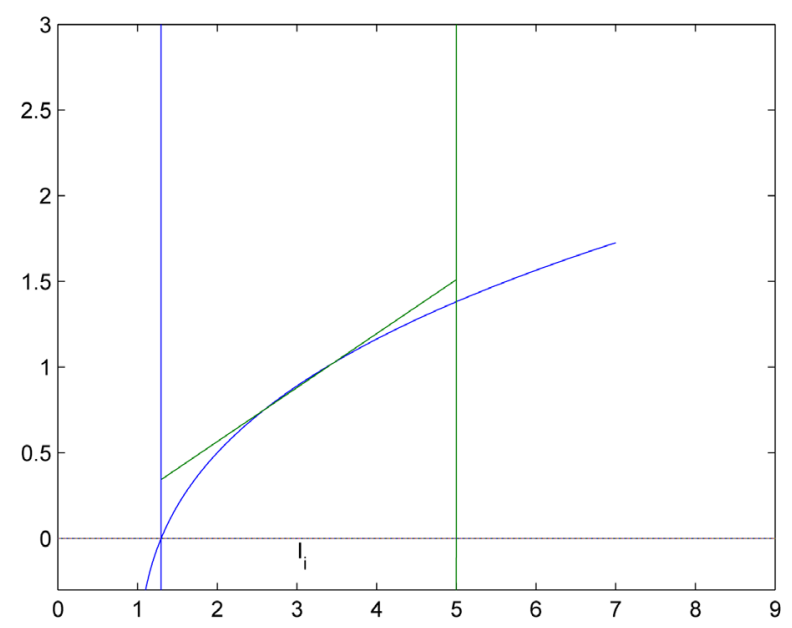

Figure 1. The line segment $a_{i} r+b_{i}$ on the interval $I_{i}$ (not drawn to scale).

\section{The Cosmological Constant}

Suppose we return to Equation (14) and substitute Equations (16)-(18). Then we obtain

$$
F^{\prime \prime}-\frac{k r}{1-k r^{2}} F^{\prime}+F\left[\omega \frac{2 k}{1-k r^{2}}+\frac{\omega+1}{r^{2}}\left(a^{2}(t) \frac{1}{1-k r^{2}}-1\right)\right]=0
$$

While we normally assume that $k \neq 0$, it is noted in Ref. [3] that $k=0$, representing a spatially flat universe, is not a dramatic departure from generality when it comes to late-time cosmology.

With $k=0$, the time-dependent solution is

$$
\begin{aligned}
F(r)= & c_{1} \exp \left[(-\ln r)\left(\frac{1}{2} \sqrt{4 \omega-4 a^{2} \omega-4 a^{2}+5}-\frac{1}{2}\right)\right] \\
& +c_{2} \exp \left[(\ln r)\left(\frac{1}{2} \sqrt{4 \omega-4 a^{2} \omega-4 a^{2}+5}+\frac{1}{2}\right)\right] .
\end{aligned}
$$

In the special case $\omega=-1, F(r)=c_{1}+c_{2} r$, in agreement with Equation (19) with $k=0$.

In the previous section, we dealt with a time-independent solution due to the assumption $\omega=-1$. This allowed our $f(R)$ modified model to remain close to Einstein gravity at least in principle. By contrast, solution (27) is time dependent. So if $\omega \neq-1$, we are dealing with two possibilities:

(a) if $\omega>-1$, there is no real solution;

(b) if $\omega<-1$, then the $f(R)$ model is far removed from Einstein gravity, i.e., if $a^{2}(t)$ increases indefinitely, then the first term in solution (27) goes to zero, while the second term gets large. So $F(r)$ cannot remain close to unity.

We conclude that $\omega=-1$ in the equation of state $p=\omega \rho$ is the only allowed value. Since this note deals with rather reasonable assumptions, the only plausible objection to this conclusion is that the equation of state for dark energy is much more complicated than the perfect-fluid equation of state $p=\omega \rho$. This possibility was also raised by Lobo [5], who stated that a mixture of various in- 
teracting non-ideal fluids may be necessary. This could imply that dark energy is dynamic in nature, thereby forcing us to exclude models with constant $\omega$, including the cosmological constant.

\section{Conclusions}

The starting point in this note is $f(R)$ modified gravity in a cosmological setting. We also assume a spatially flat universe to describe late-time cosmology [3]; thus $k=0$ in the FLRW model. Our key assumption is the perfect-fluid equation of state $p=\omega \rho$ to describe the hypothesized dark energy. While $\omega<-1 / 3$ is sufficient to yield an accelerated expansion, it is concluded that $\omega=-1$ is the only value which allows our solution to remain close enough to Einstein gravity to be consistent with observation.

Weighing the above assumptions, we conclude that either (1) Einstein's cosmological constant is the only acceptable model for dark energy, or that (2) the equation of state is far more complicated than the above perfect-fluid equation and may even exclude a constant $\omega$.

\section{References}

[1] Riess, A.G., et al. (1998) Observational Evidence from Supernovae for an Accelerating Universe and a Cosmological Constant. Astronomical Journal, 116, 1009-1038. https://doi.org/10.1086/300499

[2] Perlmutter, S.J., et al. (1999) Measurements of $\Omega$ and $\Lambda$ from 42 High-Red Shift Supernovae. The Astrophysical Journal, 517, 565-586. https://doi.org/10.1086/307221

[3] Sotiriou, T.P. and Faraoni, V. (2010) $f(R)$ Theories of Gravity. Reviews of Modern Physics, 82, 451-457. https://doi.org/10.1103/RevModPhys.82.451

[4] Nojiri, S. and Odintsov, S.D. (2007) Introduction to Modified Gravity and Gravitational Alternative for Dark Energy. International Journal of Geometric Methods in Modern Physics, 4, 115. https://doi.org/10.1142/S0219887807001928

[5] Lobo, F.S.N. (2008) The Dark Side of Gravity: Modified Theories of Gravity. arXiv: 0807.1640.

[6] Bousso, R. (2012) The Cosmological Constant Problem, Dark Energy and the Landscape of String Theory. arXiv: 1203.0307.

[7] Lobo, F.S.N. and Oliveira, M.A. (2009) Wormhole Geometries in $f(R)$ Modified Theories of Gravity. Physical Review D, 80, Article No.: 104012. https://doi.org/10.1103/physrevd.80.104012

[8] Rahaman, F., Chakraborty, K., Kuhfittig, P.K.F., Shit, G.C. and Rahman, M. (2014) A New Deterministic Model of Strange Stars. European Physical Journal C, 74, 3126. https://doi.org/10.1140/epjc/s10052-014-3126-6 
Submit or recommend next manuscript to SCIRP and we will provide best service for you:

Accepting pre-submission inquiries through Email, Facebook, LinkedIn, Twitter, etc. A wide selection of journals (inclusive of 9 subjects, more than 200 journals)

Providing 24-hour high-quality service

User-friendly online submission system

Fair and swift peer-review system

Efficient typesetting and proofreading procedure

Display of the result of downloads and visits, as well as the number of cited articles Maximum dissemination of your research work

Submit your manuscript at: http://papersubmission.scirp.org/

Or contact jamp@scirp.org 